\title{
Luigi Pagliaro, Marco Bobbio, Agostino Colli: Diagnosis in Medicine
}

\author{
1st edition, 2011, Raffaello Cortina Editore, Milan, pp 328, ISBN 978-88-6030-420-9
}

\author{
Domenico Prisco • Gian Franco Gensini
}

Published online: 19 August 2011

(C) SIMI 2011

This book, by Luigi Pagliaro, Marco Bobbio and Agostino Colli, is a very special type of medical textbook standing out of the usual format of textbooks of internal medicine.

As emphasized in the presentation written by Professor Nicola Dioguardi, this book analyzes in both logic and pragmatic terms, the most creative clinical actions performed (or not) in their everyday's practice by all clinicians.

The volume is handy and well published. It is organized according to a very logical scheme and covers a wide range of topics ranging from an historical introduction to a modern approach for a total of ten chapters:

- From diagnosis to treatment, past and present

- Pattern recognition, clinical history and physical examination

- Diagnostic tests

- Diagnostic reasoning

- How to communicate diagnosis

- Diagnostic probability and decisional thresholds

- Diagnostic studies and probability assessment

- Risk factors and diagnosis

- Errors in Medicine and errors in diagnosis

- How to learn to make diagnosis.

Although devoted to different topics, all chapters have been composed with common reproducible criteria.

The book is enriched by the presentation of 46 case records and comprehensive tables and schemes, which render it easy to read and very friendly for the reader.

D. Prisco $(\bowtie) \cdot$ G. F. Gensini

Department of Medical and Surgical Critical Care,

University of Florence, Viale Morgagni 85,

50134 Florence, Italy

e-mail: priscod@aou-careggi.toscana.it
In the preface authors remind that in medical congresses most sessions are devoted to therapeutics and just a few deal with diagnosis even if often they tend to identify tests which are more suitable to diagnose a disease. But both the strategy of diagnostic pathways and that of accumulation of several diagnostic tests are inadequate.

The Authors depict Medicine as the combination of two components: biomedicine and bio-psycho-social medicine. The latter is the background in all the volume and enters in the diagnostic process, for example when one collects medical history or communicates diagnosis. We believe that this approach really is the appropriate one, enabling to apply in the best way evidences to clinical activity through personal experience and thorough communication with the patient.

The practical aspects of the various topics are strongly emphasized, with the aim of providing a guide for medical and graduate students, residents and physicians.

In spite of being written by great experts, the book can be easily read because of its language, distant from the arid prose of classic medical science.

This book is not a rigid algorithm-based manual but rather a highly educative reading.

The writing is clear and passionate, so that one can perceive the willingness to communicate clinical problems from real day-by-day practice, conducted in close contact with patients.

Overall, this is an excellent handbook that provides a wide range of information in a complete and clear, but relatively concise, way which rigorously indicates methods and empathically transmits passion.

Gian Franco Gensini Domenico Prisco 